\title{
Haemorrhagic thyroid cyst after breathing exercise
}

Abbi Lulsegged, Mufaddal Moonim, Rose Ngu

Kings College Hospital NHS Foundation Trust, Guys and St Thomas Hospitals, London, United Kingdom

\section{Objectives:}

40-year-old gentleman

Referred with sudden onset discomfort in the neck associated with the development of a lump.

Earlier that morning he had carried out breathing exercise known as surya krishna at 6.00 a.m and noticed the lump three hours later whilst shaving.

\section{Methods:}

Thyroid USS showed a right-sided thyroid cyst.

Aspiration yielded haemorrhagic contents.

FNA of the solid component: no malignant cells.

Fluid re-accumulated with pressure symptoms, pain and heaviness in this region.

Repeat scan did not reveal any significant changes in the appearance of the thyroid cyst

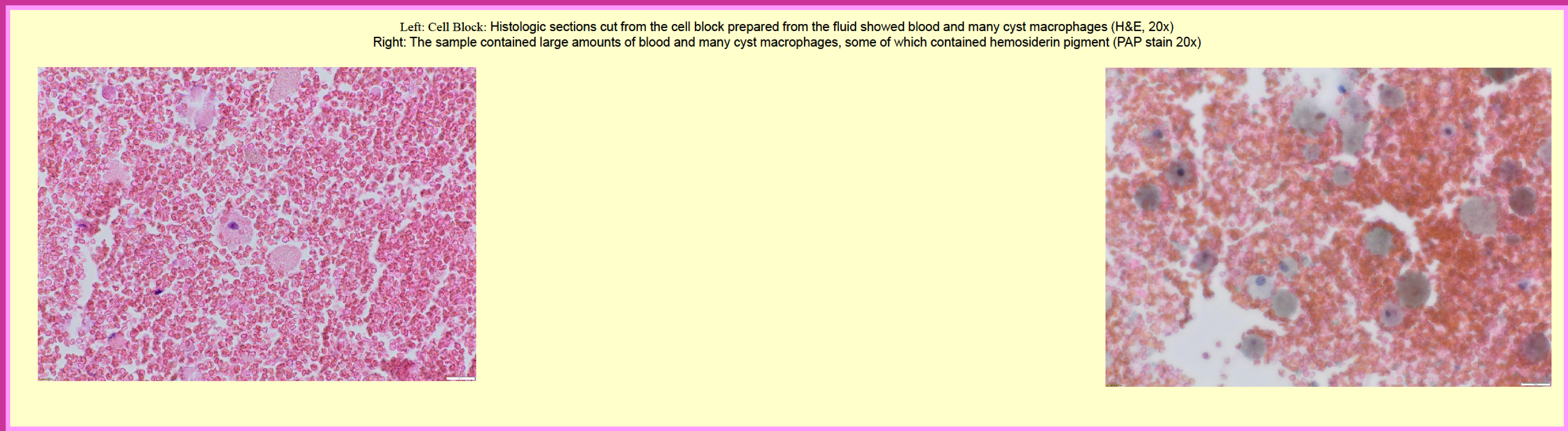

Surya krishna is a breathing exercise lasting 30-60 minutes with times of hyperventilation and other times of slow breathing. Our patient recalled experiencing pins and needles, numbness and tingling with the hyperventilation.

However at the beginning of the session there is an emphasis placed on breathing through the throat and he remembered exerting a significant pressure in the throat region when doing this.

\section{Conclusions:}

We hypothesize that the pressures involved during the breathing exercises may have been sufficient to cause haemorrhaging within a thyroid nodule.

The authors have seen 2 other cases presenting in a similar manner.

\section{References:}

Text 